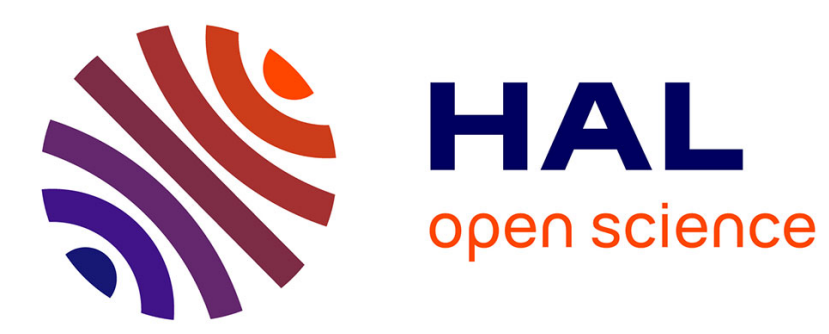

\title{
Real-time icosahedral to fcc structure transition during CoPt nanoparticle formation
}

\author{
José Penuelas, Pascal Andreazza, Caroline Andreazza-Vignolle, Christine \\ Mottet, Maurizio de Santis, Hélio C. N. Tolentino
}

\section{- To cite this version:}

José Penuelas, Pascal Andreazza, Caroline Andreazza-Vignolle, Christine Mottet, Maurizio de Santis, et al.. Real-time icosahedral to fcc structure transition during CoPt nanoparticle formation. European Journal of Physics, 2009, 167, pp.19-25. 10.1140/epjst/e2009-00931-x . hal-00443733

\section{HAL Id: hal-00443733 \\ https://hal.science/hal-00443733}

Submitted on 4 Jan 2010

HAL is a multi-disciplinary open access archive for the deposit and dissemination of scientific research documents, whether they are published or not. The documents may come from teaching and research institutions in France or abroad, or from public or private research centers.
L'archive ouverte pluridisciplinaire HAL, est destinée au dépôt et à la diffusion de documents scientifiques de niveau recherche, publiés ou non, émanant des établissements d'enseignement et de recherche français ou étrangers, des laboratoires publics ou privés. 
EPJ manuscript No.

(will be inserted by the editor)

\title{
Real-time icosahedral to fcc structure transition during CoPt nanoparticle formation
}

\author{
J. Penuelas ${ }^{1}$, P. Andreazza ${ }^{1, a}$, C. Andreazza-Vignolle ${ }^{1}$, C. Mottet $^{2}$, M. De Santis ${ }^{3}$, and H.C.N. \\ Tolentino ${ }^{3}$ \\ 1 Centre de Recherche sur la Matière Divisée, UMR 6619 Université d'Orléans-CNRS, 1 bis, rue de la \\ Férollerie, 45071 Orléans Cedex, France \\ 2 Centre Interdisciplinaire des Nanosciences de Marseille, CNRS, Campus de Luminy, case 913, 13288 \\ Marseille Cedex 9, France \\ 3 Insitut Néel, CNRS \& UFJ, 25 avenue des Martyrs BP 166, 38042 Grenoble Cedex 9, France
}

\begin{abstract}
Nucleation and growth of supported CoPt nanoparticles were studied in situ and in real time by combined grazing incidence small-angle x-ray scattering (GISAXS) and grazing incidence x-ray diffraction (GIXD). GISAXS provides morphological features of nanoparticles as size, shape and correlation distance between nanoparticles, while GIXD allows the determination of the atomic structure. In this study we focused on the formation of ultrasmall CoPt nanoparticles, in the $1-4 \mathrm{~nm}$ size range. A structural analysis method based on the Debye equation is presented coupled with cluster model calculations. These Monte Carlo simulations of relaxed CoPt cluster structures (disordered truncated octahedral, decahedral and icosahedral) were performed using a semi-empirical tight-binding potential to interpret diffraction spectra and structural transition. The experiment was performed at $500^{\circ} \mathrm{C}$ in UHV conditions at the European Synchrotron Radiation Facility (ESRF) on the BM32 SUV beamline. Our results show that the evolution of the cluster structure during the growth is size-dependent and coalescence-dependent. In the early stage $(N<300$ atoms $)$ of CoPt growth, coalescence events are the mainly responsible to the fcc nanoparticle formation, from icosahedral morphology.
\end{abstract}

\section{Introduction}

Supported transition-metal nanoparticles (NPs) present growing interest in many areas of material science because of their catalytic [1], optical and magnetic properties [2]. In the nanometer scale, the particle size plays a significant role in their structures and as a consequence in their chemical and physical properties. The size-dependent evolutions of the structure and structural transitions of nanoparticles have been the subject of fundamental and applied research interests [3]. Thus, the equilibrium structure of a nanometer-sized particle may differ from the bulk. In the case of most transition metals, the atomic structure is based on the spheres packing in a densest arrangement, i.e. a perfect tetrahedral packing (face-centered cubic or hexagonal close-packed). However, ultrasmall particles with icosahedral or decahedral structures were commonly observed since the pioneer works of Ino [4] on gold. These non-crystalline structures are composed of distorted tetrahedral units where adjacent tetrahedral faces are connected by a twinning plane. The resulting morphologies favor the low energy (111)-like close-packed facets and induce large internal strains. These non-crystalline morphologies exhibit fivefold

\footnotetext{
a e-mail: Pascal.Andreazza@univ-orleans.fr
} 
symmetry incompatible with the translational symmetry of crystalline lattices. Their stability depends on a critical balance between surface and volume contributions to the total energy and consequently decreases as the size increases. The icosahedral morphology should be the most favored at small size, while fcc morphologies like truncated octahedron are expected for larger sizes; decahedra could be favored in the intermediate range [3]. In the case of bimetallic nanoparticles, not only the size but also the composition can influence their structures [5-7]. The classical sequence of stability could be modified by segregation and finite size effects. In addition, kinetic processes involved during their elaboration can induce metastable structures such as large icosahedra or decahedra $[8,9]$ as well as metastable chemical arrangements such as three-shell onionlike bimetallic NPs [10]. The existence of such particles suggests that the conditions under which their growth occurred have led to non-equilibrium structures. In addition to kinetic effects, surface contamination and contact effect with external support or matrix have been identified as factors inducing a change of the particle structure and morphology [11, 12]. As a consequence, the knowledge of the NPs formation mechanisms is of prime importance since their properties are directly connected to their structure.

In this article, we focus on morphological and structural investigations of supported CoPt NPs in the ultrasmall size range $1<$ diameter $<4 \mathrm{~nm}$. The case of CoPt system is particularly interesting due to the high structural sensitivity of the magnetic properties of CoPt equi-atomic composition nanoparticles $[13,14]$. We used surface sensitive x-ray scattering methods as Grazing Incidence Small-Angle X-ray Scattering (GISAXS) and Grazing Incidence X-ray Diffraction (GIXD) to follow in situ and in real-time the nucleation and growth processes. As compared to Transmission Electron Microscopy (TEM) [15] which is a technique restricted to the particle observation in a limited area, GIXD and GISAXS techniques probe complementarily the order and morphology at the atomic scale of nano-objects on a statistical average over the whole sample. In addition, due to the high surface to volume ratio and the high reactivity of Co, in situ analysis under ultrahigh vaccum (UHV) is absolutely required to prevent an influence of the environment on the morphology or structure of nanoparticles, even in the case of clusters protected by a coating or embedded in a matrix. The real-time aspect of the experiment is of particular interest to study dynamical processes during each stage of the growth (nucleation, growth and coalescence). Many studies by small- and/or wide-angle scattering have been done for monometallic clusters as $\mathrm{Au}$ [16-18] or Ag [19]. To our knowledge few papers present bimetallic NPs x-ray scattering investigations in real-time and in situ conditions. We report in this paper the temperature effect during the growth, in term of atoms and particles mobility and in term of structural evolution. The choice of a high growth temperature $\left(500{ }^{\circ} \mathrm{C}\right)$ was motivated by the interest to enhance atoms mobility and consequently the structural rearrangement. A structural analysis method based on the Debye equation is presented coupled with cluster model calculations. These Monte Carlo simulations of relaxed CoPt cluster structures (disordered truncated octahedral, decahedral and icosahedral) were performed using a semi-empirical tight-binding potential to interpret diffraction spectra and structural transition. Through these investigations, we identify specific morphologies and structures and follow the size-dependent and temperature-dependent evolutions of the chemically disordered CoPt NPs.

\section{Experimental}

The experiments were performed at BM32 beamline located at the European Radiation Synchrotron Facility (ESRF) using the UHV setup allowing molecular beam epitaxy growth combined to in situ and in real time GISAXS and GIXD measurements [20,21]. Figure 1(a) shows a schematic view of the experiment geometry. The two-dimensional GISAXS pattern was recorded using a CCD camera mounted at a sample-detector distance of $1 \mathrm{~m}$. Due to the much higher scattering intensity at $\mathrm{q}_{\|}=0$ because of the specularly reflected beam and the diffuse reflectivity in comparison to the GISAXS signal at finite values for $\mathrm{q}_{\|}$, both tungsten beam-stop and aluminum absorber foils were used in order to optimally match the measured intensity with the given dynamic range of the CCD detector. A standard scintillator detector, which could be positioned in the scattering direction (fixed exit angle $\alpha_{f}=\alpha_{i}$ and scan angle $\delta$ ), was used for the GIXD intensities measurements. This GIXD geometry ensures that the length of the beam 
(a)
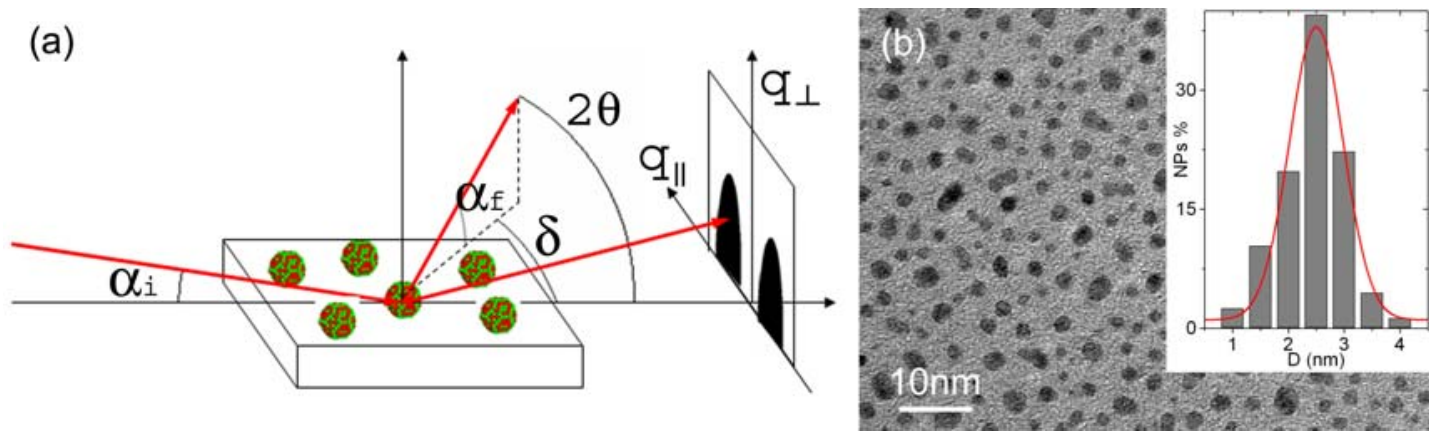

Fig. 1. (a) Schematic drawing of the scattering measurements geometry. Monochromatic x-ray beam of $18 \mathrm{keV}$ were irradiated on the sample at a grazing angle $\alpha_{i}$ of $0.07^{\circ}$. The scattered intensity is recorded as a function of the in-plane angle $\alpha_{f}$ with respect to the surface plane and of the in-plane angle $\delta$. These angles allow defining the reciprocal space coordinates $\mathrm{q}_{\perp}$ and $\mathrm{q}_{\|}$, respectively perpendicular and parallel to the sample surface. (b) TEM micrograph of NPs CoPt sample annealed at $500{ }^{\circ} \mathrm{C}$. The inset displays the size distribution histogram.

path within the sample, which is relevant for absorption, is constant during the measurements. In both cases, due to the low size range of particles and low quantities of deposited metal, the $\mathrm{x}$-ray grazing incidence angle were selected in order to reduce the scattering background from amorphous substrate.

Co and Pt atoms were co-deposited by condensation in the UHV chamber using electron beam evaporation on prepared substrate. The base pressure was $1 \times 10^{-10} \mathrm{mbar}$, this value increased to $3 \times 10^{-10}$ mbar during the deposition. The substrates used for cluster condensation were amorphous $\mathrm{SiO}_{2}$ coated with amorphous carbon (a-C) layer of $10 \mathrm{~nm}$. These substrates were chosen to limit the cluster-substrate interactions and to allow comparisons with our previous TEM results obtained on a-C deposited nanoparticles [15]. Substrates were degassed at $650^{\circ} \mathrm{C}$ with a ramp rate of $10^{\circ} \mathrm{C} / \mathrm{min}$ to vaporize water and some organic species. Co and $\mathrm{Pt}$ deposition rates were calibrated to obtain a global composition close to $\mathrm{Co}_{50} \mathrm{Pt}_{50}$ with constant flux during all growth stages. These rates $\left(0.26 \times 10^{15}\right.$ atoms $/ \mathrm{cm}^{2} /$ hour $)$ were selected as weak to limit the kinetic effects on the bimetallic NPs during the growth. The temperature fixed to $500^{\circ} \mathrm{C}$ was calibrated by a thermocouple and a pyrometer during the deposition.

\section{Results and discussion}

\subsection{Modeling and analysis procedure}

The morphological parameters of the nanoparticles as diameter $D$, height $H$ and correlation distance between particles $\Lambda$ were extracted from GISAXS experimental data by comparison with simulations using IsGISAXS software [22] in the Distorded Wave Born Approximation. The GISAXS intensity of an assembly of islands is proportional to the form factor of the nanoparticles and the interference function, which is the Fourier transform of the NP position autocorrelation function. For a size distribution system as observed in TEM [Fig. 1(b)], the local monodisperse approximation could be applied in the simulation, then the total scattered intensity was calculated as the incoherent summation of the intensities of domains with monodisperse size. For the form factor, the best results were obtained with a spherical shape that can be truncated by taking an aspect ratio $H / D<1$. A narrow Gaussian size distribution with constant relative geometrical standard deviation $\sigma(D) / D=0.3$ according to our previous TEM results [15] was used for each simulation. In order to analyze 2D patterns [see Fig. 2(a)], two cuts of the intensity in the $\mathrm{q}_{\|}$and $\mathrm{q}_{\perp}$ directions (determined by $\delta$ and $\alpha_{f}$, respectively) are simultaneously fitted to determine the evolution of the dimensional parameters [19]. Preliminary to the GISAXS analysis, we performed ex-situ TEM observations on a sample prepared in the same UHV set-up and with same deposition parameters (Fig. 1(b)). The substrate was 

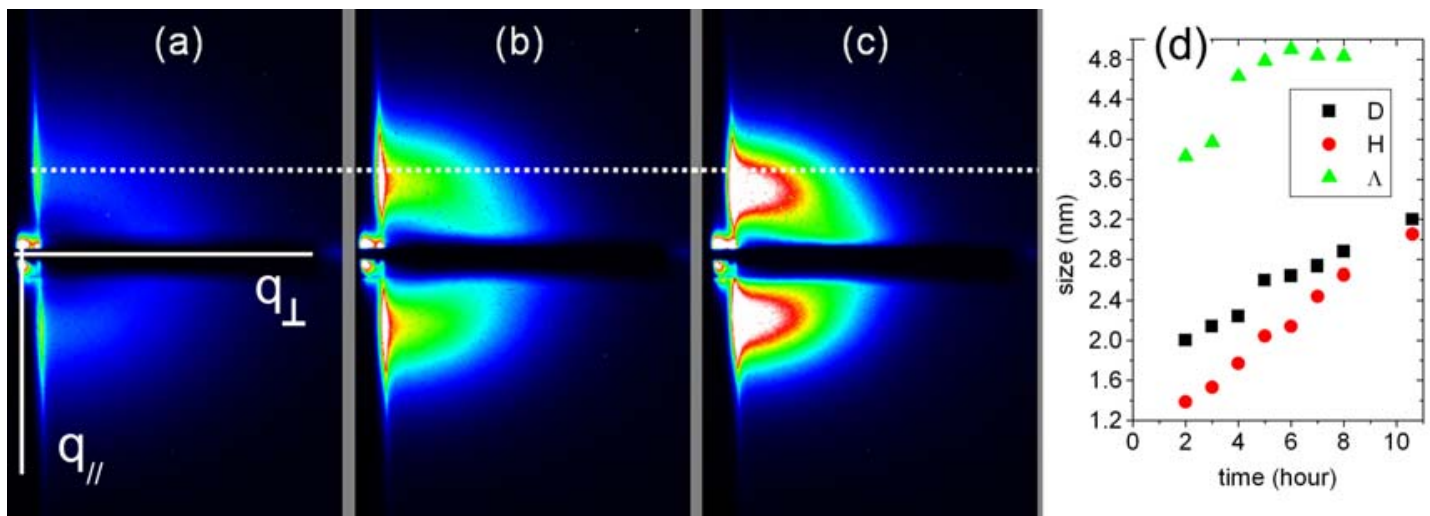

Fig. 2. Measured GISAXS patterns of sample grown at $500^{\circ} \mathrm{C}$ at different growth times: (a) 3, (b) 6 and (c) 9 hours. The white dotted line is a visual guide line to show the shift of the interference function maximum. (d) graph shows the evolution of diameter $D$, height $H$ and correlation distance between particles $\Lambda$, extracted from GISAXS experimental data ( $\mathrm{q}_{\perp}$ and $\mathrm{q}_{\|}$cross sections).

a self-supported amorphous carbon membrane prepared in the same conditions than the top layer of the silica substrate. Statistical measurements gave an average diameter of $2.5 \mathrm{~nm}$ with a Gaussian size distribution in accordance with selected parameters given above.

The diversity of particle structures obtained in nanometer scale as described in the Introduction don't allow a simple identification from diffraction data as in a bulk material. One alternative is to calculate the wide angle scattering pattern of predicted finite structures of nanoparticles in a size range deduced from GISAXS data analysis. The scattering intensity for a model cluster is calculated quantitatively on the basis of the Debye equation $[23,24,26]$ from a list of the coordinates of all atoms (Co and Pt types) contained in each kind of structure. Polarization evolution effects, preliminary calculated, were neglected due to their weak evolution with $q$. We have used three types of structures in our cluster models: truncated octahedral (TOh) based on face-centered-cubic structure, decahedral (Dh) and icosahedral (Ih) according respectively with Wulff [27], Marks [28] and Ino [4] constructions. To determine the atomic structure of our samples, we used either single size and single cluster type to fit the diffraction patterns, or a weighted sum of the intensities from several sizes or structures [24]. Models composed of clusters with complete shells were found to be sufficient for data analysis in a size range between 50 and 2000 atoms according to GISAXS results. Monte Carlo (MC) simulations of equiconcentration $\mathrm{CoPt}$ cluster structures (TOh. Dh and Ih illustrated in the insets of Fig. 4) were performed using a semi-empirical tight-binding potential [29]. The models are relaxed at a temperature (between 410 and $550 \mathrm{C}$ ) close to the experimental value $\left(500^{\circ} \mathrm{C}\right)$. Earlier studies [30] have shown that the use of pure geometrical structures induces significant changes in the scattering pattern as important as particle size decreases. Figure 3(a) illustrates this tendency in the case of a fcc TOh model of geometric and MC relaxed nanoparticles of 405 atoms (around $2 \mathrm{~nm}$ in size). The variation of atom positions in the simulated structure with respect to geometric model is due to structural and thermal relaxations that must be taken into account in the case of NPs. In addition, Figure 3(b) shows the evolution of broad diffraction peaks located at Bragg angles of TOh fcc crystalline models, which become sharper with increasing cluster size (in the $1.5-3 \mathrm{~nm}$ size range). Finally, the wide-angle scattering profiles of the three different structures (TOh, Dh and Ih) for a similar size $(2.5 \mathrm{~nm})$ are clearly distinguishable (see Figure 3(c)) and thus, allow the discrimination between the different models [24]. The diffraction technique is rather sensible to the symmetry than to the size or to the local atomic distortions. 

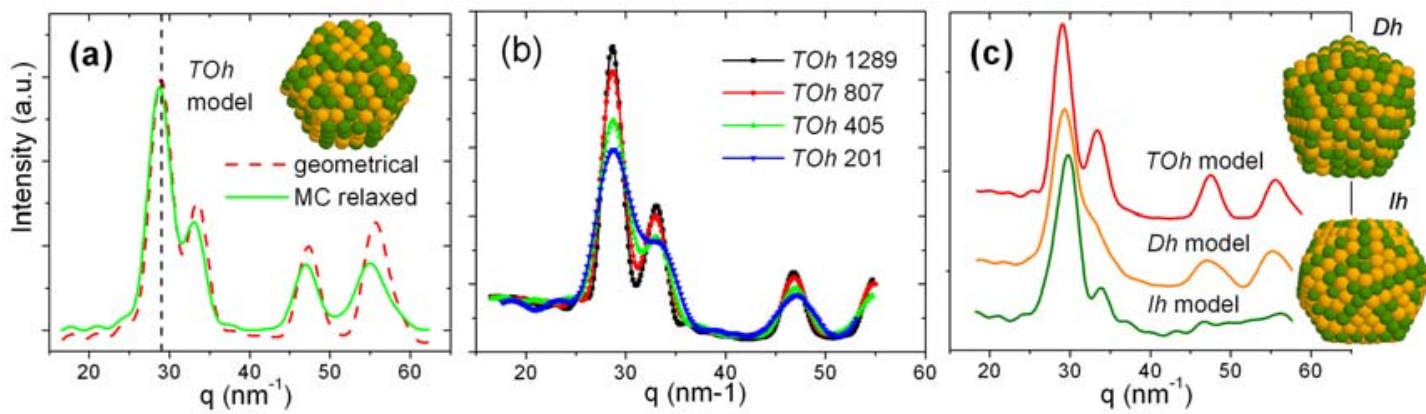

Fig. 3. Calculated GIXD spectra of (a) geometric and Monte Carlo relaxed (TOh) truncated octahedron CoPt clusters of 405 atoms (2nm),(b) a series of CoPt TOh clusters of increasing size, 201, 405, 807 and 1289 atoms corresponding to 1.5, 2, 2.5 and $3 \mathrm{~nm}$ in diameter, (c) TOh, decahedral (Dh) and icosahedral (Ih) models of clusters around $2.5 \mathrm{~nm}$ in diameter. The insets shows the snapshots of different MC simulated clusters (Co and Pt atoms are represented as light and dark spheres respectively).

\subsection{GISAXS and GIXD analysis}

Real-time GISAXS measurements were performed during the deposition on amorphous carbon surface heated at $500{ }^{\circ} \mathrm{C}$ with a frame interval of 30 minutes. Figure 2 shows the representative evolution of 2D patterns which contain informations on the size, the shape and the correlation distance of the nanoparticles. The 2D GISAXS patterns present two lobes of scattered intensity which are already observed from one hour of deposition. However, a quantitative analysis was possible only from $2 \mathrm{~h}$ of deposition time. The progressive shift of the maximal intensity position to the low $\mathrm{q}_{\|}$values and the lobe extension (Fig. 2(a-c)) indicate an evolution of the form factor and of the interference function, simultaneously. From this evolution with time, we deduced the evolution of $D$ and $H$ shown in Figure 2(d). These parameters increase with the deposition time according to theoretical predictions in the case of Volmer Weber growth. The NPs diameter and height $(D, H)$ of truncated sphere shape is found to increase from $(2 \mathrm{~nm}, 1.4 \mathrm{~nm})$ at $2 \mathrm{~h}$ deposition time to $(3.2 \mathrm{~nm}, 3.1 \mathrm{~nm})$ at the end of the deposition experiment (Fig. 2(d)). The faster increasing of height compared to the diameter is classically observed for high coverage rate on the substrate surface, when a direct capture of atoms on the NPs top is favored [31]. In our case, the coverage rate remains low (15-20\%). Thus, this shape evolution from a flat to isotropic shape is certainly due to the high substrate temperature. This temperature favor the atom mobility in the clusters which leads to an equilibrium morphology as the experiment duration increase. Concerning the mean inter-particle distance $\Lambda$, the value remains quasi constant from $4 \mathrm{~h}$ deposition time until the end of the experiment. At earlier stages of the growth, the results suggest that after a nucleation stage corresponding to a $\Lambda$ decreasing, a dynamic coalescence occurs corresponding to the increasing of the mean inter-particle distance (between 2 and $4 \mathrm{~h}$ ).

Simultaneously with GISAXS investigations, diffraction patterns were obtained during the CoPt deposition (Fig. 4(a)) showing the scattering contribution of the nanoparticles. The "background" pattern displays mainly the scattering contribution of the amorphous substrate. To obtain the scattered signal due to the deposited metal, the data were normalized (see examples in Fig. 4(b)) taking into account the absorption effect in the substrate and the background subtracted [32]. Thus, the resulting metal contribution (see fig. 4(c-f))) can be compared with simulated GIXD spectra to provide a fit of the CoPt experimental patterns. For the highest deposition times (Fig. 4(a)), the diffraction patterns features are in agreement with the different peaks of the fcc structure with an interatomic distance of $2.65 \AA$, close to the A1 CoPt bulk value, proving the alloying of $\mathrm{Co}$ and $\mathrm{Pt}$. This experimental value is consistent with the MC simulated model $(2.68 \AA$ ). Indeed, this latest value correspond also to the interatomic distance obtained by Monte Carlo simulation in bulk phase with the same interatomic potential [29]. At the early stages of the deposition, the results are not in agreement with a fcc crystalline structure but rather with a non-crystalline atomic arrangement. For the medium time deposition (5h), Fig. 4(c), (d) and (e) show the superposition between calculated and experimental 

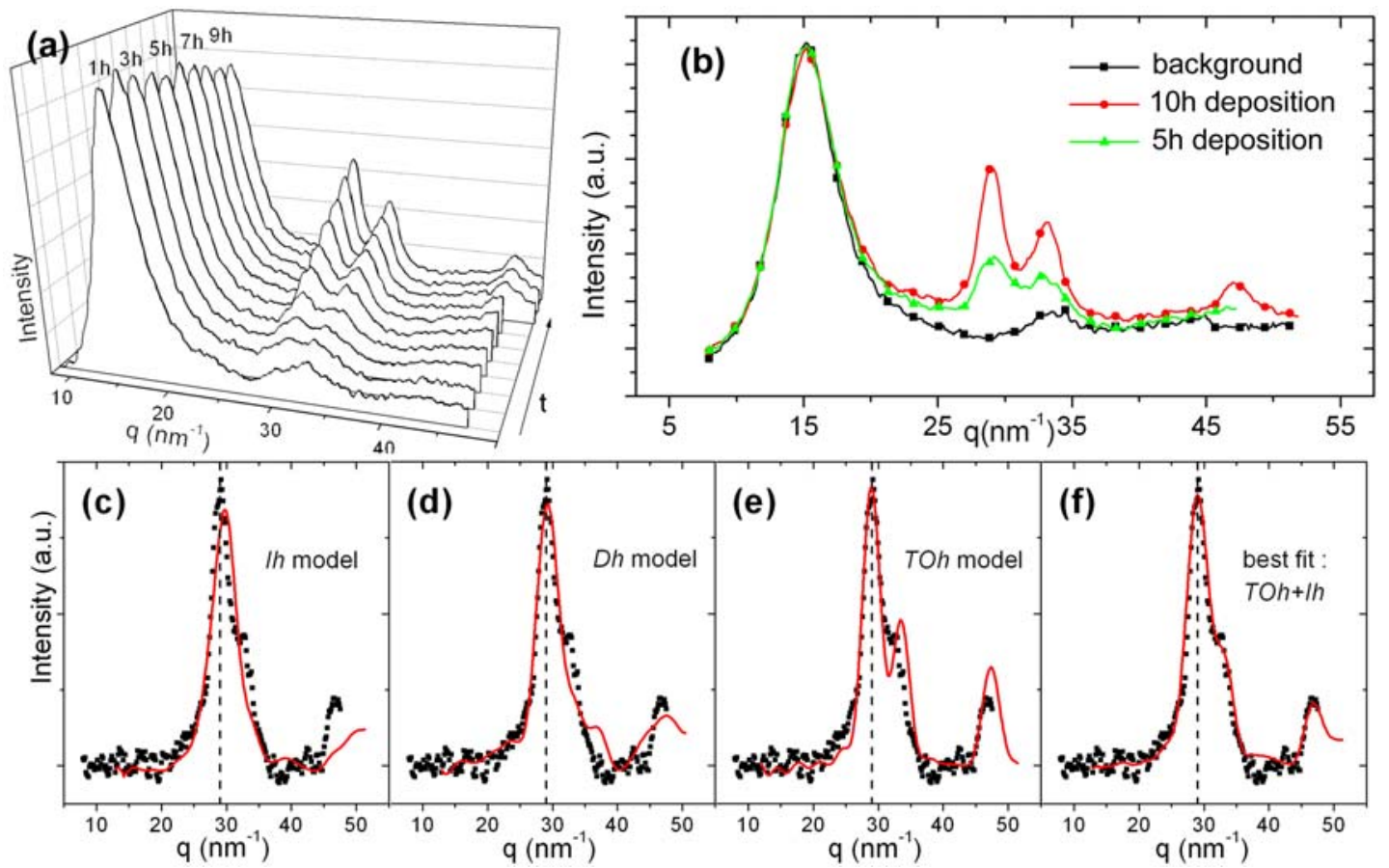

Fig. 4. (a) Evolution of the GIXD patterns as a function of time ( $\mathrm{t}$ in hours) from 1 to $10 \mathrm{~h}$. (b) Three examples of normalized patterns compared with background obtained at $500^{\circ} \mathrm{C}$ before the deposition. (c), (d), (e) comparison between NPs contribution experimental pattern at $5 \mathrm{~h}$ deposition time and calculated patterns of relaxed $\mathrm{Ih}, \mathrm{Dh}$ and TOh models, respectively. (f) best fit obtained with a mix of Ih and TOh models

patterns of the three different models. Icosahedral, Decahedral and fcc models were fitted to the data without a good agreement. The best fit is obtained with a mix of Ih and TOh-fcc models (around 50\% each in atomic fraction) with $\mathrm{N}$ around 309 and 405 atoms respectively. These results suggest a continuous structure transition, from Ih to TOh structure, only due to a size effect and the clusters have reached their equilibrium shape during the growth.

According to semi-empirical calculations [3], on pure Pt and Co metal clusters, as sizes increase the icosahedral range is dropped in favor of decahedral structures, and approaching to the bulk limit the fcc structure (TOh) becomes the most favorable. In the case of pure Pt clusters, for example, stable decahedra cover a size range for which lower limit is placed at $N \sim 75$. In the case of pure Co, the decahedral range starts at $N \sim 400$. In the CoPt disordered equi-atomic case [29], the calculation results indicate that the Dh range begins at $N \sim 100$. As regards to the transition from the Dh to fcc motif, calculations suggest that decahedra and fcc models are competing already at $N \sim 600$, so the transition could take place in the $600<N<1000$ size range. Our results showing a structural evolution with the NPs size, are consistent with these tight binding simulations but without Dh structure formation in a intermediate size range, as previously observed in pure cluters [25]. However, the icosahedral structure observed at the early stage of NPs formation shows a Ih stability at larger size $N \sim 400$ than predicted by equilibrium calculations. Our experiments were performed with a low deposition rate, but nevertheless during the deposition under non equilibrium conditions. In addition, the GISAXS results concerning the evolution of the shape and interparticle distance suggest a coalescence process during the early stage of the growth. Such events can affect the structure by making highly defective clusters, with morphological disruption and large number of stacking faults [33] to allow the NPs transformation to the minimum energy morphology, i.e. the TOh shapes at larger sizes [34]. After the coalescence stage, the fcc resulting morphology was locked in and the size increase is dominated by atom by atom growth mechanism. 


\section{Conclusion}

To conclude, we have shown that combined GISAXS and GIXD techniques can allow a fine morphological and structural analysis of CoPt nanoparticles in the $1-4 \mathrm{~nm}$ size range. However, these experiments must be supported by wide angle scattering calculations using cluster models. Thus, Monte Carlo simulations of relaxed CoPt cluster structures (disordered truncated octahedral, decahedral and icosahedral) were performed using a semi-empirical tight-binding potential, to interpret diffraction pattern. Real time scattering experiments were performed during the formation of supported equi-atomic composition CoPt nanoparticles obtained by UHV deposition. Our results show that in the nanoparticles assembly, icosahedral structure dominates at early stage (small particle sizes) of the growth process and fcc structure appears at larger sizes. The structural change occurs because of competition between surface and volume energy components as predicted by MC simulations. However, this structural transition was favored by the coalescence between small icosahedral nanoparticles.

\section{References}

1. C. R. Henry, Surf. Sci. Rep. 31, 235 (1998)

2. J. Bansmann et al, Surf. Sci. Rep. 56, 189 (2005)

3. F. Baletto, R. Ferrando, Rev. Mod. Phys. 77, 371 (2005)

4. S. Ino, J. Phys. Soc. Jpn. 27, 941 (1969)

5. B. Pauwels, G. Van Tendeloo, E. Zhurkin, M. Hou, G. Verschoren, L. Theil Kuhn, W. Bouwen, P. Lievens, Phys. Rev. B 63, 165406 (2001)

6. C. Mottet, G. Treglia, and B. Legrand, Phys. Rev. B 66, 045413 (2002)

7. S. Giorgio and C. Henry, Microsc. Microanal. Microstruct. 8, 379 (1997)

8. J. L. Rodriguez-Lopez, J. M. Montejano-Carrizales, U. Pal, J. F. Sanchez-Ramirez, H. E. Troiani, D. Garcia, M. Miki-Yoshida, M. Jose-Yacaman, Phys. Rev. Lett. 92, 196102 (2004)

9. R. M. Wang, O. Dmitrieva, M. Farle, G. Dumpich, H. Q. Ye, H. Poppa, R. Kilaas, and C. Kisielowski Phys. Rev. Lett. 100, 017205 (2008)

10. F. Baletto, C. Mottet, R. ferrando, Phys. Rev. Lett. 90, 135504 (2003).

11. L. Favre et al., Phys. Rev. B 74, 014439 (2006)

12. S. Anders, M. F. Toney, T. Thomson, R. F. C. Farrow, J.-U. Thiele, B. D. Terris, Shouheng Sun, and C. B. Murray, J. Appl. Phys. 93, 6299 (2003)

13. F. Tournus, A. Tamion, N. Blanc, A. Hannour, L. Bardotti, B. Prvel, P. Ohresser, E. Bonet, T. Epicier, and V. Dupuis, Phys. Rev. B 77, 144411 (2008)

14. P. Imperia, P. Andreazza, D. Schmitz, J. Penuelas, C. Andreazza-Vignolle, J. Magnetism. Mag. Mat. 310, 2417 (2007)

15. J. Penuelas, C. Andreazza-Vignolle, P. Andreazza, A. Ouerghi, N. Bouet, Surf. Sci. 602, 545 (2008)

16. S. Narayanan, D. Ryeol. Lee, R. S. Guico, S. K. Sinha, J. Wang, Phys. Rev. Lett. 94, 145504 (2005)

17. K. Koga, H. Takeo, T. Ikeda, K. I. Ohshima, Phys. Rev. B 57, 4053 (1998)

18. B. Abécassis, F. Testard, O. Spalla, P. Barboux, Nanoletters 7, 1723 (2007)

19. C. Revenant, G. Renaud, R. Lazzari, J. Jupille, Nucl. Instr. and Meth. in Phys. Res. B 246, 112 (2006)

20. R. Baudoing-Savois et al., Nucl. Instr. and Meth. in Phys. Res. B, 149, 213 (1999)

21. G. Renaud, et al., Science 300, 1416 (2003)

22. R. Lazzari, J. Appl. Cryst. 35, 406 (2002).

23. B. E. Warren, X-Ray Diffraction (Dover, New York, 1990)

24. D. Reinhard, B. D. Hall, P. Berthoud, S. Valkealahti, R. Monot, Phys. Rev. Lett. 79, 1459 (1997)

25. D. Reinhard, B. D. Hall, D. Ugarte, R. Monot, Phys. Rev. B 55, 7868 (1997)

26. J. Penuelas, P. Andreazza, C. Andreazza-Vignolle, H. C. N. Tolentino, M. De Santis, and C. Mottet Phys. Rev. Lett. 100, 115502 (2008)

27. C.L. Cleveland and U. Landman J. Chem. Phys. 94, 7376 (1991)

28. L. D. Marks, Phil. Mag. A 49, 81 (1984)

29. G. Rossi, R. Ferrando, C. Mottet, Faraday Discuss. 138, 193 (2008)

30. B.D. Hall, J. Appl. Phys. 88, 1666 (2000)

31. M. Zinke-Allmang, L. C. Feldman, M. H. Grabow, Surf. Sci. Rep. 16, 377 (1992).

32. V. Kiryukhin, E. P. Bernard, V. V. Khmelenko, R. E. Boltnev, N. V. Krainyukova, D. M. Lee, Phys. Rev. Lett. 98, 195506 (2007)

33. S. Hendy, S. A. Brown, M. Hyslop, Phys. Rev. B 68, 241403 (2003).

34. G. Grochola, S. P. Russo, I. K. Snook, J. Chem. Phys. 126, 164707 (2007). 